\title{
NEURAL NETWORKS WITH DISTRIBUTED DELAYS AND HÖLDER CONTINUOUS ACTIVATION FUNCTIONS
}

\author{
NASSER-EDDINE TATAR
}

Received 21 March, 2014

\begin{abstract}
We consider a system which arises in Neural Network Theory with distributed delays involving Hölder continuous activation functions. We prove some results on global exponential stability of the system. This extends the previous works where activation functions were assumed to be Lipschitz continuous.
\end{abstract}

2010 Mathematics Subject Classification: 24G20; 34C11; 92B20

Keywords: neural network, distributed delay, exponential stability, Hölder continuity, non-Lipschitz continuity

\section{INTRODUCTION}

In this work we investigate the following system

$$
x_{i}^{\prime}(t)=-a_{i}(t) x_{i}(t)+\sum_{j=1}^{m} b_{i j} \int_{-\infty}^{t} K_{i j}(t-s) f_{j}\left(x_{j}(s)\right) d s+c_{i}(t), i=1, \ldots, m
$$

for $t>0$, with $x_{j}(t)=x_{0 j}(t), t \in(-\infty, 0]$ where $x_{0 j}(t)$ are given continuous functions and $b_{i j}$ are real constants. The functions $a_{i}(t)$ (nonnegative), $c_{i}(t), i=1, \ldots, m$ are continuous functions and $f_{j}$ are the activation functions. The functions $K_{i j}(t)$ are assumed to be continuous and integrable.

This system arises in (Artificial) Neural Network theory [7,8] in which there is a growing interest these last two decades. Unlike the conventional machines (based on von Neumann architecture) which use a single processor, the Neural Network consists of a large number of processors (arranged in layers) and in general a larger number of interconnections between them. A processor receives inputs from the preceding layer, process it and then pass it to the subsequent layer. There are various applications in: engineering, science, biology, finance, medicine and geology. Neural Networks are used to understand (complex) phenomena in these fields. They are able

The author is grateful for the financial support and the facilities provided by King Fahd University of Petroleum and Minerals through project No. IN121044. 
to classify and recognize patterns. They are also used to solve mathematical programming problems. Their advantages over the traditional computers are the ability to perform huge parallel computations, to classify certain products and materials and to predict certain phenomena.

There are many papers in the literature dealing with the local or global asymptotic stability of the system. In particular, we are witnessing a lot of interest in exponential stability of the (unique) equilibrium of the system for this problem and other similar ones $[2,3,15-18,21,23,25,26,28]$ to cite but a few. A lot of efforts are devoted in improving the set of conditions on the different coefficients involved in the system as well as the class of activation functions. For the coefficients the main conditions turn around a kind of dominance of the "dissipation" coefficients $a_{i}$ on the other coefficients. As for the activation functions the first researchers have dealt with specific explicit ones. Then they moved to the assumptions: monotonicity, differentiability and boundedness. These conditions were later weakened to a Lipschitz condition. After that not much has been done for continuous but not Lipschitz continuous activation functions compared to the Lipschitz case or even the discontinuous case. NonLipschitz continuous activation functions arise in many fields [10], see also [6] for an application involving Hölder continuous functions. For a slightly weaker condition or partial Lipschitz condition we refer the reader to [3, 4, 23, 25].

Hölder continuous activation functions were studied by Forti et al. in [4] for the problem

$$
x^{\prime}=B x+T g(x)+I .
$$

The authors assumed that each component of $g$ is bounded and is a non-decreasing piecewise continuous function. The matrix $-T$ is assumed Lyapunov Diagonally Stable. An exponential stability result and a finite time convergence result is proved there and extended to a larger class of non-Lipschitz continuous activation functions under a stronger condition. In addition to that the authors investigated discontinuous activation functions using the theory of Filippov. In fact discontinuous activation functions have been treated in several other papers (see [1,5,9,12-14, 19, 20, 22, 24]) first under some boundedness and monotonicity conditions. Later these condition were dropped under some other conditions.

Here we consider variable coefficients and activation functions $f_{j}$ that are Hölder continuous i.e.

$$
\left|f_{j}(x)-f_{j}(y)\right| \leq L_{j}|x-y|^{\alpha_{j}}, 0<\alpha_{j}<1
$$

for some positive constants $L_{j}, j=1, \ldots, m$. Clearly, Hölder continuous functions are not necessarily Lipschitz continuous. We prove global exponential stability of the system. This is achieved with the help of a Gronwall-type inequality due to E. H. Yang [27], some appropriate estimates and some Lyapunov-type functionals.

The local existence is standard whereas the global existence may be derived using (2.2) and the Gronwall-type Lemma 1 below.

In the next section we state and prove our results. 


\section{EXPONENTIAL STABILITY}

In this section it is proved that the system is globally asymptotically stable in an exponential manner when the activation functions (all or some of them) are only Hölder continuous.

Definition 1. We say that the system (1.1) is globally asymptotically stable if for any two solutions $x_{i}(t)$ and $\bar{x}_{i}(t)$ we have

$$
\lim _{t \rightarrow \infty} \sum_{i=1}^{m}\left|x_{i}(t)-\bar{x}_{i}(t)\right|=0 .
$$

It is said to be exponentially asymptotically stable if there exist two positive constants $M$ and $\eta$ such that

$$
\sum_{i=1}^{m}\left|x_{i}(t)-\bar{x}_{i}(t)\right| \leq M e^{-\eta t}, t>0 .
$$

In case the coefficients in problem (1.1) are constant and there exists a unique equilibrium $x_{i}^{*}, i=1, \ldots, m$, that is a solution of

$$
0=-a_{i} x_{i}^{*}+\sum_{j=1}^{m} b_{i j} f_{j}\left(x_{j}^{*}\right) \int_{0}^{\infty} K_{i j}(s) d s+c_{i}, i=1, \ldots, m .
$$

then we obtain exponential stability of this equilibrium.

We denote by $y_{i}(t)=x_{i}(t)-\bar{x}_{i}(t), y(t)=\sum_{i=1}^{m}\left|y_{i}(t)\right|, a(t):=\min _{1 \leq i \leq m}\left\{a_{i}(t)\right\}$,

$$
\phi_{1}(t):=a(t)-\sum_{i, j=1}^{m} \frac{L_{j}\left|b_{i j}\right|}{p_{j}} \mu_{j}(t) \int_{0}^{\infty}\left|K_{i j}(s)\right| e^{\int_{t}^{t+s} a(\sigma) d \sigma} d s, t \geq 0
$$

and

$$
\chi_{1}(t):=\sum_{i, j=1}^{m} \frac{L_{j}\left|b_{i j}\right|}{q_{j}} \int_{-\infty}^{t}\left|K_{i j}(t-s)\right| \mu_{j}^{-q_{j} / p_{j}}(s) d s, t \geq 0
$$

for some positive continuous functions $\mu_{j}(t), p_{j}=1 / \alpha_{j}$ and $q_{j}=1 /\left(1-\alpha_{j}\right), j=$ $1, \ldots, m$.

Theorem 1. Assume that $f_{j}, j=1, \ldots, m$ are Hölder continuous and $K_{i j}(t)$, $i, j=1, \ldots, m$ are continuous and integrable functions. Assume further that $a_{i}(t)$, $b_{i j}(t), L_{j}, i, j=1, \ldots, m$ and the continuous functions $\mu_{i}(t)>0$ are such that $\phi_{1}(t) \geq 0$ (not identically zero), $\int_{0}^{t} \phi_{1}(\sigma) d \sigma \rightarrow \infty$ as $t \rightarrow \infty$ and

$$
\int_{0}^{t} \chi_{1}(s) e^{\int_{0}^{s} \phi_{1}(\sigma) d \sigma} d s
$$

is at most of polynomial growth $P(t)$. Then, the system (1.1) globally asymptotically stable at the rate $P(t) e^{-\int_{0}^{t} \phi_{1}(s) d s}$, that is

$$
y(t) \leq P(t) e^{-\int_{0}^{t} \phi_{1}(s) d s}, t>0 .
$$


Proof. From the system (1.1), the assumptions (1.2), (2.2) and the adopted notation we get

$$
D^{+}\left|y_{i}(t)\right| \leq-a_{i}(t)\left|y_{i}(t)\right|+\sum_{j=1}^{m} L_{j}\left|b_{i j}\right| \int_{-\infty}^{t}\left|K_{i j}(t-s)\right|\left|y_{j}(s)\right|^{\alpha_{j}} d s, t>0,
$$

for $i=1, \ldots, m$, where $D^{+}$denotes the right Dini derivative. Therefore,

$$
D^{+} y(t) \leq-\min _{1 \leq i \leq m}\left\{a_{i}(t)\right\} y(t)+\sum_{i, j=1}^{m} L_{j}\left|b_{i j}\right| \int_{-\infty}^{t}\left|K_{i j}(t-s)\right|\left|y_{j}(s)\right|^{\alpha_{j}} d s, t>0
$$

Hence, as we denote $a(t):=\min _{1 \leq i \leq m}\left\{a_{i}(t)\right\}$, we get

$$
D^{+} y(t) \leq-a(t) y(t)+\sum_{i, j=1}^{m} L_{j}\left|b_{i j}\right| \int_{-\infty}^{t}\left|K_{i j}(t-s)\right|\left|y_{j}(s)\right|^{\alpha_{j}} d s, t>0 .
$$

We estimate $|y(t)|^{\alpha_{i}}, 0<\alpha_{i}<1, i=1, \ldots, m$ using Young's inequality as follows

$$
\left|y_{j}(t)\right|^{\alpha_{j}} \leq \frac{\mu_{j}(t)}{p_{j}}\left|y_{j}(t)\right|+\frac{\mu_{j}^{-q_{j} / p_{j}}(t)}{q_{j}}, \mu_{j}(t)>0, \frac{1}{p_{j}}+\frac{1}{q_{j}}=1, j=1, \ldots, m, t \geq 0
$$

with $p_{j}=1 / \alpha_{j}$ and $q_{j}=1 /\left(1-\alpha_{j}\right), j=1, \ldots, m$. Plugging these relations (2.3) in (2.2) we find

$$
\begin{aligned}
D^{+} y(t) \leq & -a(t) y(t) \\
& +\sum_{i, j=1}^{m} L_{j}\left|b_{i j}\right| \int_{-\infty}^{t}\left|K_{i j}(t-s)\right|\left[\frac{\mu_{j}(s)}{p_{j}}\left|y_{j}(s)\right|+\frac{\mu_{j}^{-q_{j} / p_{j}}(s)}{q_{i}}\right] d s \\
\leq & -a(t) y(t)+\sum_{i, j=1}^{m} \frac{L_{j}\left|b_{i j}\right|}{p_{j}} \int_{-\infty}^{t}\left|K_{i j}(t-s)\right| \mu_{j}(s)\left|y_{j}(s)\right| d s \\
& +\sum_{i, j=1}^{m} \frac{L_{j}\left|b_{i j}\right|}{q_{j}} \int_{-\infty}^{t}\left|K_{i j}(t-s)\right| \mu_{j}^{-q_{j} / p_{j}}(s) d s, t>0 .
\end{aligned}
$$

Now we introduce the following functional

$$
V_{1}(t):=y(t)+\Phi_{1}(t), t \geq 0
$$

where

$$
\Phi_{1}(t):=\sum_{i, j=1}^{m} \frac{L_{j}\left|b_{i j}\right|}{p_{j}} e^{-\int_{0}^{t} a(s) d s} \int_{0}^{\infty}\left|K_{i j}(s)\right| \int_{t-s}^{t} e^{\int_{0}^{\tau+s} a(\sigma) d \sigma} \mu_{j}(\tau)\left|y_{j}(\tau)\right| d \tau d s .
$$


This functional $\Phi_{1}(t)$ will help us get rid of the delayed terms. Indeed, by differentiation it is easy to see that

$$
\begin{aligned}
& \Phi_{1}^{\prime}(t):=-a(t) \Phi_{1}(t)+\sum_{i, j=1}^{m} \frac{L_{j}\left|b_{i j}\right|}{p_{j}} e^{-\int_{0}^{t} a(s) d s} \\
& \times \int_{0}^{\infty}\left|K_{i j}(s)\right|\left[e^{\int_{0}^{t+s} a(\sigma) d \sigma} \mu_{j}(t)\left|y_{j}(t)\right|-e^{\int_{0}^{t} a(\sigma) d \sigma} \mu_{j}(t-s)\left|y_{j}(t-s)\right|\right] d s \\
& =-a(t) \Phi_{1}(t)+\sum_{j=1}^{m}\left[\sum_{i=1}^{m} \frac{L_{j}\left|b_{i j}\right|}{p_{j}} \int_{0}^{\infty}\left|K_{i j}(s)\right| e^{\int_{t}^{t+s} a(\sigma) d \sigma} d s\right] \mu_{j}(t)\left|y_{j}(t)\right| \\
& \quad-\sum_{i, j=1}^{m} \frac{L_{j}\left|b_{i j}\right|}{p_{j}} \int_{0}^{\infty}\left|K_{i j}(s)\right| \mu_{j}(t-s)\left|y_{j}(t-s)\right| d s, t>0
\end{aligned}
$$

where the last term is equal to the second term in the right hand side of (2.4) with an opposite sign. So summing up (2.4) and (2.6) we get

$$
\begin{aligned}
D^{+} V_{1}(t) \leq & -a(t) y(t)-a(t) \Phi_{1}(t) \\
& +\sum_{i, j=1}^{m} \frac{L_{j}\left|b_{i j}\right|}{p_{j}} \int_{-\infty}^{t}\left|K_{i j}(t-s)\right| \mu_{j}(s)\left|y_{j}(s)\right| d s \\
& +\sum_{i, j=1}^{m} \frac{L_{j}\left|b_{i j}\right|}{q_{j}} \int_{-\infty}^{t}\left|K_{i j}(t-s)\right| \mu_{j}^{-q_{j} / p_{j}}(s) d s \\
& +\sum_{j=1}^{m}\left[\sum_{i=1}^{m} \frac{L_{j}\left|b_{i j}\right|}{p_{j}} \int_{0}^{\infty}\left|K_{i j}(s)\right| e^{\int_{t}^{t+s} a(\sigma) d \sigma} d s\right] \mu_{j}(t)\left|y_{j}(t)\right| \\
& -\sum_{i, j=1}^{m} \frac{L_{j}\left|b_{i j}\right|}{p_{j}} \int_{0}^{\infty}\left|K_{i j}(s)\right| \mu_{j}(t-s)\left|y_{j}(t-s)\right| d s \\
\leq & -\left[a(t)-\sum_{i, j=1}^{m} \frac{L_{j}\left|b_{i j}\right|}{p_{j}} \mu_{j}(t) \int_{0}^{\infty}\left|K_{i j}(s)\right| e^{\int_{t}^{t+s}} a(\sigma) d \sigma d s\right] V_{1}(t) \\
& +\sum_{i, j=1}^{m} \frac{L_{j}\left|b_{i j}\right|}{q_{j}} \int_{-\infty}^{t}\left|K_{i j}(t-s)\right| \mu_{j}^{-q_{j} / p_{j}}(s) d s, t>0 .
\end{aligned}
$$

With our notation we can rewrite (2.7) as

$$
D^{+} V_{1}(t) \leq-\phi_{1}(t) V_{1}(t)+\chi_{1}(t), t>0 .
$$

We derive that

$$
D^{+}\left\{V_{1}(t) e^{\int_{0}^{t} \phi_{1}(s) d s}\right\} \leq \chi_{1}(t) e^{\int_{0}^{t} \phi_{1}(s) d s}, t>0 .
$$


A standard comparison theorem (see Theorem 1.4.1 in [11]) implies that

$$
V_{1}(t) e^{\int_{0}^{t} \phi_{1}(s) d s} \leq V_{1}(0)+\int_{0}^{t} \chi_{1}(s) e^{\int_{0}^{s} \phi_{1}(\sigma) d \sigma} d s, t>0
$$

or

$$
V_{1}(t) \leq V_{1}(0) e^{-\int_{0}^{t} \phi_{1}(s) d s}+e^{-\int_{0}^{t} \phi_{1}(s) d s} \int_{0}^{t} \chi_{1}(s) e^{\int_{0}^{s} \phi_{1}(\sigma) d \sigma} d s, t>0 .
$$

The assumption that

$$
\int_{0}^{t} \chi_{1}(s) e^{\int_{0}^{s} \phi_{1}(\sigma) d \sigma} d s
$$

is at most of polynomial growth implies that $V_{1}(t)$ that and therefore $y(t)$ is decaying exponentially to zero.

The following lemma due to E. H. Yang [27] will be needed in our next result.

Lemma 1. Let $u(t)$ and $f_{i}(t) i=1, \ldots, n$ be non-negative continuous functions on an interval $J=[0, T), 0<T \leq 0$ and $a(t)$ a non-decreasing function such that $a(t) \geq 1$ for $t \in J$. If

$$
u(t) \leq a(t)+\sum_{i=1}^{n} \int_{0}^{t} f_{i}(s)(u(s))^{r_{i}} d s, t \in J, 0<r_{i}<1
$$

then

$$
u(t) \leq a(t) \prod_{i=1}^{n} G_{i}(t), t \in J
$$

where

$$
G_{i}(t):=\left[1+\left(1-r_{i}\right) \prod_{k=1}^{i-1} G_{k}(t) \int_{0}^{t} f_{i}(s) d s\right]^{\frac{1}{2-r_{i}}}, i=1, \ldots, n
$$

and $\prod_{i=1}^{0} G_{i}(t)=1, t \in J$.

In our present situation we will use

$$
G_{i}(t):=\left[1+\left(1-\alpha_{i}\right) \prod_{k=1}^{i-1} G_{k}(t) \int_{0}^{t} \tilde{b}_{i}(s) d s\right]^{\frac{1}{2-\alpha_{i}}}, i=1, \ldots, m
$$

where

$$
\tilde{b}_{i}(t):=\left[\sum_{i=1}^{m} L_{j}\left|b_{i j}\right| \int_{0}^{\infty}\left|K_{i j}(s)\right| e^{\int_{0}^{s+t} a(\sigma) d \sigma} d s\right] e^{-\alpha_{j} \int_{0}^{t} a(\sigma) d \sigma} .
$$

Theorem 2. Assume that $f_{j}$ are Hölder continuous i.e. satisfy the relations (1.2), $j=1, \ldots, m, \quad K_{i j}(t), i, j=1, \ldots, m$ are continuous and integrable functions, 
$\prod_{i=1}^{m} G_{i}(t)$ grows at most as a polynomial and $\int_{0}^{t} a(s) d s \rightarrow \infty$ as $t \rightarrow \infty$. Then, we have

$$
y(t) \leq[1+y(0)] \prod_{i=1}^{m} G_{i}(t) \exp \left(-\int_{0}^{t} a(s) d s\right), t>0 .
$$

Proof. We start from the inequality (see (2.2))

$$
D^{+} y(t) \leq-a(t) y(t)+\sum_{i, j=1}^{m} L_{j}\left|b_{i j}\right| \int_{-\infty}^{t}\left|K_{i j}(t-s)\right|\left|y_{j}(s)\right|^{\alpha_{j}} d s, t>0 .
$$

Consider the new functional

$$
V_{2}(t):=y(t)+\Phi_{2}(t), t \geq 0
$$

where

$$
\Phi_{2}(t):=\sum_{i, j=1}^{m} L_{j}\left|b_{i j}\right| e^{-\int_{0}^{t} a(s) d s} \int_{0}^{\infty}\left|K_{i j}(s)\right| \int_{t-s}^{t} e^{\int_{0}^{s+\tau} a(\sigma) d \sigma}\left|y_{j}(\tau)\right|^{\alpha_{j}} d \tau .
$$

A differentiation of $\Phi_{2}(t)$ in (2.10) gives

$$
\begin{aligned}
\Phi_{2}^{\prime}(t)= & -a(t) \Phi_{2}(t)+\sum_{i, j=1}^{m} L_{j}\left|b_{i j}\right| e^{-\int_{0}^{t} a(s) d s} \int_{0}^{\infty}\left|K_{i j}(s)\right| e^{\int_{0}^{s+t} a(\sigma) d \sigma} d s\left|y_{j}(t)\right|^{\alpha_{j}} \\
& -\sum_{i, j=1}^{m} L_{j}\left|b_{i j}\right| \int_{0}^{\infty}\left|K_{i j}(s)\right|\left|y_{j}(t-s)\right|^{\alpha_{j}} d s, t \geq 0
\end{aligned}
$$

and from (2.8)-(2.11) we see that

$$
\begin{aligned}
D^{+} V_{2}(t) \leq & -a(t) y(t)-a(t) \Phi_{2}(t)+\sum_{i, j=1}^{m} L_{j}\left|b_{i j}\right| \int_{-\infty}^{t}\left|K_{i j}(t-s)\right|\left|y_{j}(s)\right|^{\alpha_{j}} d s \\
& +\sum_{i, j=1}^{m} L_{j}\left|b_{i j}\right| e^{-\int_{0}^{t} a(s) d s} \int_{0}^{\infty}\left|K_{i j}(s)\right| e^{\int_{0}^{s+t} a(\sigma) d \sigma} d s\left|y_{j}(t)\right|^{\alpha_{j}} \\
& -\sum_{i, j=1}^{m} L_{j}\left|b_{i j}\right| \int_{0}^{\infty}\left|K_{i j}(s)\right|\left|y_{j}(t-s)\right|^{\alpha_{j}} d s \\
\leq & -a(t) V_{2}(t) \\
& +\sum_{j=1}^{m}\left[\sum_{i=1}^{m} L_{j}\left|b_{i j}\right| e^{-\int_{0}^{t} a(s) d s} \int_{0}^{\infty}\left|K_{i j}(s)\right| e^{\int_{0}^{s+t} a(\sigma) d \sigma} d s\right] V_{2}^{\alpha_{j}}(t), t>0 .
\end{aligned}
$$

Thus

$$
D^{+}\left[V_{2}(t) \exp \int_{0}^{t} a(s) d s\right]
$$




$$
\begin{aligned}
\leq & \sum_{j=1}^{m}\left[\sum_{i=1}^{m} L_{j}\left|b_{i j}\right| \int_{0}^{\infty}\left|K_{i j}(s)\right| e^{\int_{0}^{s+t} a(\sigma) d \sigma} d s\right] V_{2}^{\alpha_{j}}(t) \\
\leq & \sum_{j=1}^{m}\left[\sum_{i=1}^{m} L_{j}\left|b_{i j}\right| \int_{0}^{\infty}\left|K_{i j}(s)\right| e^{\int_{0}^{s+t} a(\sigma) d \sigma} d s\right]\left[\exp \left(-\alpha_{j} \int_{0}^{t} a(s) d s\right)\right] \\
& \times\left[V_{2}(t) \exp \int_{0}^{t} a(s) d s\right]^{\alpha_{j}}, t>0
\end{aligned}
$$

and by comparison and integration

$$
\begin{aligned}
V_{2}(t) \exp \int_{0}^{t} a(s) d s \leq & V_{2}(0)+\int_{0}^{t}\left\{\sum_{j=1}^{m}\left[\sum_{i=1}^{m} L_{j}\left|b_{i j}\right| \int_{0}^{\infty}\left|K_{i j}(\tau)\right| e^{\int_{0}^{\tau+s} a(\sigma) d \sigma} d \tau\right]\right. \\
& \left.e^{-\alpha_{j} \int_{0}^{s} a(\sigma) d \sigma}\left[V_{2}(s) \exp \int_{0}^{s} a(\sigma) d \sigma\right]^{\alpha_{j}}\right\} d s, t>0 .
\end{aligned}
$$

In short

$$
\tilde{V}_{2}(t) \leq V_{2}(0)+\sum_{j=1}^{m} \int_{0}^{t} \tilde{b}_{j}(s)\left[\tilde{V}_{2}(s)\right]^{\alpha_{j}} d s, t>0
$$

where $\tilde{V}_{2}(t):=V_{2}(t) \exp \int_{0}^{t} a(s) d s$ and

$$
\tilde{b}_{i}(t):=\left[\sum_{i=1}^{m} L_{j}\left|b_{i j}\right| \int_{0}^{\infty}\left|K_{i j}(\tau)\right| e^{\int_{0}^{s+t} a(\sigma) d \sigma} d \tau\right] e^{-\alpha_{j} \int_{0}^{t} a(\sigma) d \sigma}, i=1, \ldots, m .
$$

Therefore we can apply Lemma 1 to obtain

$$
\tilde{V}_{2}(t) \leq\left[1+V_{2}(0)\right] \prod_{j=1}^{m} G_{j}(t)
$$

where

$$
G_{j}(t):=\left[1+\left(1-\alpha_{j}\right) \prod_{k=1}^{j-1} G_{k}(t) \int_{0}^{t} \tilde{b}_{j}(s) d s\right]^{\frac{1}{2-\alpha_{j}}}, j=1, \ldots, m
$$

and $\prod_{i=1}^{0} G_{i}(t)=1, t \geq 0$. The proof is complete.

Remark 1. The coefficients $b_{i j}$ being assumed constants is only a technical assumption to avoid duplication of an undesirable term in the derivative of $\Phi_{2}(t)$.

\section{REFERENCES}

[1] G. Bao and Z. Zeng, "Analysis and design of associative memories based on recurrent neural network with discontinuous activation functions," Neurocomputing, vol. 77, no. 1, pp. 101-107, 2012, doi: 10.1016/j.neucom.2011.08.026.

[2] J. Cao, K. Yuan, and H.-X. Li, "Global asymptotical stability of recurrent neural networks with multiple discrete delays and distributed delays," IEEE Trans. Neural Netw., vol. 17, no. 6, pp. 1646-1651, 2006, doi: 10.1109/tnn.2006.881488. 
[3] C. Feng and R. Plamondon, "On the stability analysis of delayed neural networks systems," Neural Networks, vol. 14, no. 9, pp. 1181-1188, 2001, doi: 10.1016/s0893-6080(01)00088-0.

[4] M. Forti, M. Grazzini, P. Nistri, and L. Pancioni, "Generalized Lyapunov approach for convergence of neural networks with discontinuous or non-Lipschitz activations," Physica D: Nonlinear Phenomena, vol. 214, no. 1, pp. 88-99, 2006, doi: 10.1016/j.physd.2005.12.006.

[5] M. Forti and P. Nistri, "Global convergence of neural networks with discontinuous neuron activations," IEEE Trans. Circuits Syst.-I: Fund. Theory Appl., vol. 50, no. 11, pp. 1421-1435, 2003, doi: $10.1109 /$ tcsi.2003.818614.

[6] R. Gavaldà and H. T. Siegelmann, "Discontinuities in recurrent neural networks," Neural Comput., vol. 11, no. 3, pp. 715-745, 1999, doi: 10.1162/089976699300016638.

[7] J. J. Hopfield, "Neural networks and physical systems with emergent collective computational abilities," Proc. Nat. Acad. Sci., vol. 79, no. 8, pp. 2554-2558, 1982, doi: 10.1142/9789812799371-0043.

[8] J. J. Hopfield, D. W. Tank et al., "Computing with neural circuits- a model," Science, vol. 233, no. 4764, pp. 625-633, 1986, doi: 10.1126/science.3755256.

[9] Y. Huang, H. Zhang, and Z. Wang, "Dynamical stability analysis of multiple equilibrium points in time-varying delayed recurrent neural networks with discontinuous activation functions," Neurocomputing, vol. 91, pp. 21-28, 2012, doi: 10.1016/j.neucom.2012.02.016.

[10] B. Kosko, Neural Network and Fuzzy System - A Dynamical System Approach to Machine Intelligence. Prentice-Hall, New-Delhi,, 1991.

[11] V. Lakshmikantham and S. Leela, Differential and integral inequalities. Academic press, 1969.

[12] L. Li and L. Huang, "Dynamical behaviors of a class of recurrent neural networks with discontinuous neuron activations," Appl. Math Model., vol. 33, no. 12, pp. 4326-4336, 2009, doi: 10.1016/j.apm.2009.03.014.

[13] J. Liu, X. Liu, and W.-C. Xie, "Global convergence of neural networks with mixed time-varying delays and discontinuous neuron activations," Information Sciences, vol. 183, no. 1, pp. 92-105, 2012, doi: 10.1016/j.ins.2011.08.021.

[14] X. Liu and J. Cao, "Robust state estimation for neural networks with discontinuous activations," IEEE Trans. Syst., Man, Cyb.-Part B: Cybernetics, vol. 40, no. 6, pp. 1425-1437, 2010, doi: 10.1109/tsmcb.2009.2039478.

[15] X. Liu and N. Jiang, "Robust stability analysis of generalized neural networks with multiple discrete delays and multiple distributed delays," Neurocomputing, vol. 72, no. 7, pp. 1789-1796, 2009, doi: 10.1016/j.neucom.2008.06.005.

[16] S. Mohamad, K. Gopalsamy, and H. Akca, "Exponential stability of artificial neural networks with distributed delays and large impulses," Nonlinear Anal., Real World Appl., vol. 9, no. 3, pp. 872-888, 2008, doi: 10.1016/j.nonrwa.2007.01.011.

[17] J. H. Park, "On global stability criterion of neural networks with continuously distributed delays," Chaos, Solitons \& Fractals Chaos Solitons \& Fractals, vol. 37, no. 2, pp. 444-449, 2008, doi: 10.1016/j.chaos.2006.09.021.

[18] Z. Qiang, M. Run-Nian, and X. Jin, "Global exponential convergence analysis of Hopfield neural networks with continuously distributed delays," Commun. Theor. Phys., vol. 39, no. 3, pp. 381384, 2003, doi: 10.1088/0253-6102/39/3/381.

[19] S. Qin and X. Xue, "Global exponential stability and global convergence in finite time of neural networks with discontinuous activations," Neural Process Lett., vol. 29, no. 3, pp. 189-204, 2009, doi: 10.1007/s11063-009-9103-7.

[20] J. Wang, L. Huang, and Z. Guo, "Global asymptotic stability of neural networks with discontinuous activations," Neural Networks, vol. 22, no. 7, pp. 931-937, 2009, doi: 10.1016/j.neunet.2009.04.004. 
[21] Y. Wang, W. Xiong, Q. Zhou, B. Xiao, and Y. Yu, "Global exponential stability of cellular neural networks with continuously distributed delays and impulses," Physics Letters A, vol. 350, no. 1, pp. 89-95, 2006, doi: 10.1016/j.physleta.2005.10.084.

[22] Z. Wang, L. Huang, Y. Zuo, and L. Zhang, "Global robust stability of time-delay systems with discontinuous activation functions under polytopic parameter uncertainties," Bull. Korean Math. Soc., vol. 47, no. 1, pp. 89-102, 2010, doi: 10.4134/bkms.2010.47.1.089.

[23] H. Wu, "Global exponential stability of Hopfield neural networks with delays and inverse Lipschitz neuron activations," Nonlinear Anal., Real World Appl., vol. 10, no. 4, pp. 2297-2306, 2009, doi: 10.1016/j.nonrwa.2008.04.016.

[24] H. Wu, "Global stability analysis of a general class of discontinuous neural networks with linear growth activation functions," Information Sciences, vol. 179, no. 19, pp. 3432-3441, 2009, doi: 10.1016/j.ins.2009.06.006.

[25] H. Wu, F. Tao, L. Qin, R. Shi, and L. He, "Robust exponential stability for interval neural networks with delays and non-Lipschitz activation functions," Nonlinear Dynamics, vol. 66, no. 4, pp. 479487, 2011, doi: 10.1007/s11071-010-9926-9.

[26] H.-F. Yanai and S.-I. Amari, "Auto-associative memory with two-stage dynamics of nonmonotonic neurons," IEEE Trans. Neural Netw., vol. 7, no. 4, pp. 803-815, 1996, doi: 10.1109/72.508925.

[27] E.-H. Yang, "Perturbations of nonlinear systems of ordinary differential equations," J. Math. Anal. Appl., vol. 103, no. 1, pp. 1-15, 1984, doi: 10.1016/0022-247x(84)90151-3.

[28] J. Zhou, S. Li, and Z. Yang, "Global exponential stability of Hopfield neural networks with distributed delays," Appl. Math. Model., vol. 33, no. 3, pp. 1513-1520, 2009, doi: 10.1016/j.apm.2008.02.006.

\section{Author's address}

\section{Nasser-eddine Tatar}

King Fahd University of Petroleum and Minerals, Department of Mathematics and Statistics, Dhahran, 31261 City, Saudi Arabia

E-mail address: tatarn@kfupm.edu.sa 